\title{
PREDAÇÃO EM CAPÍTULOS DE PICÃO-PRETO E SUA RELAÇÃO COM O TAMANHO E O GRAU DE AGRUPAMENTO DAS PLANTAS ${ }^{1}$
}

\author{
YURIKO A. N. P. YANAGIZAWA ${ }^{2}$, ADRIANA O. FIDALGO ${ }^{3}$ E RITA C. S. MAIMONI-RODELLA ${ }^{2}$
}

\section{RESUMO}

Com o objetivo de verificar possíveis correlações entre níveis de predação de capítulos de B. pilosa e o tamanho das plantas, bem como com o seu grau de agrupamento, o presente trabalho foi desenvolvido em áreas ruderais nos arredores da cidade de Botucatu, SP. Em cada coleta, foram obtidos 15 indivíduos em fase reprodutiva, sendo dez deles provenientes de agrupamentos e cinco isolados, no período de março a setembro de 1993, totalizando seis coletas. Cada planta foi caracterizada quanto a parâmetros biométricos por meio de mensurações, contagens e determinação da biomassa das diferentes estruturas, avaliando-se também a ocorrência de predação nos capítulos. Nas duas condições de agrupamento, o tamanho das plantas foi altamente variável havendo, porém, maior freqüência nas menores classes de tamanho. De modo geral, não houve diferença significativa entre plantas agrupadas e isoladas no que se refere aos parâmetros biométricos analisados. Plantas maiores produziram maior número de capítulos e o nível de predação correlacionou-se positivamente com o tamanho das plantas, independentemente do grau de agrupamento das populações. A distribuição agrupada não condicionou, portanto, maiores níveis de predação, uma vez que plantas maiores dos dois grupos foram preferencialmente atacadas. Isto corrobora a Hipótese do Vigor proposta para explicar relações de preferência entre plantas e seus herbívoros.

Palavras-chave: Bidens pilosa, herbivoria, relações de preferência.

\section{ABSTRACT \\ Bidens pilosa flower heads predation in relation to plant size and grouping}

The correlation among B. pilosa flower heads predation and plant size and grouping degree was investigated in ruderal areas at Botucatu, SP, southeastern Brazil. Fifteen plants in reproductive stage were collected monthly, from March to September, 1993. At each collection, ten plants were harvested from grouped and five from isolated plants. The plants were evaluated in relation to biometrics characteristics, biomassa distribution and flower head predation. Plant size showed variation, and the higher frequencies were observed in the smaller size classes. Isolated and grouped plants had no difference in relation to mostly of the biometrics characteristics. A greater number of flower heads was found in bigger plants which exhibited also greater predation level in both isolated and grouped individuals. Therefore grouped and isolated plants showed similar predation levels. Bigger plants, in both types of spatial distribution, had greater number of predated flower heads. This result corroborates the Vigor Hypothesis concerning preference relation between plants and herbivores.

Key words: Bidens pilosa, herbivory, preference relations.

\footnotetext{
1 Recebido para publicação em 09/03/99 e na forma revisada em 22/02/2000.

2 Prof. Ass. Dr., IBB/UNESP, Distrito de Rubião Jr, C.P. 510, CEP: 18618-000, Botucatu/SP.

${ }^{3}$ Bióloga, ex -estagiária do Departamento de Botânica, IBB/UNESP.
} 


\section{INTRODUÇÃO}

O consumo de sementes por animais representa um tipo de predação verdadeira (Begon et al., 1996) cujo efeito imediato é a redução do número de sementes viáveis disponíveis para as próximas gerações. No entanto, nem toda a predação de sementes interfere na regulação populacional pois níveis baixos ou moderados de ataque podem ser suportados pelas plantas, sem que isto venha a afetar significativamente o tamanho da população (Harper, 1977). A manutenção de uma fauna de insetos predadores nos capítulos de espécies da família Asteraceae, que constituem populações numerosas e amplamente distribuídas, descrita qualitativamente por Needhan (1946), Steyskal (1972) e Lewinsohn (1991), parece atestar este fato.

Bidens pilosa, planta daninha de ampla ocorrência no Brasil (Leitão Filho et al., 1975; Lorenzi, 1982) é muito freqüente em ambientes ruderais e agrícolas na região de Botucatu, SP (Pagliarini, 1991). Nesta mesma região já foram registradas algumas espécies de dípteros cuja forma larval consome as sementes dessas plantas, bem como himenópteros parasitóides destes predadores (Neder, 1983).

A ocorrência e a intensidade de predação podem estar associadas a uma série de características das populações vegetais hospedeiras, dentre as quais se destacam o tamanho e o grau de agrupamento das plantas. Várias hipóteses procuram explicar estes fenômenos que ainda permanecem, em parte, pouco compreendidos em decorrência da multiplicidade de fatores envolvidos (Crawley, 1997).

Com relação ao tamanho das plantas, duas hipóteses foram formuladas: a Hipótese do Estresse e a Hipótese do Vigor. De acordo com a primeira, plantas em estresse fisiológico seriam preferencialmente atacadas (White, 1969), enquanto a segunda, admite que isto ocorreria nas plantas mais vigorosas da população (Price, 1991). Quanto ao grau de agrupamento, considera-se que a distribuição espacial agrupada produz maior concentração de recursos (Altieri, 1988) atraindo, conseqüentemente, maior quantidade de herbívoros

Observações preliminares sobre a distribuição espacial de $B$. pilosa na região de Botucatu, SP, mostraram a ocorrência de plantas agrupadas e plantas isoladas na mesma área e, em ambas as situações, acentuada variação no tamanho dos indivíduos. Com base no exposto, o presente trabalho foi realizado para avaliar possíveis variações nos níveis de predação em função do tamanho e do grau de agrupamento das plantas de B. pilosa.

\section{MATERIAL E MÉTODOS}

Indivíduos de $B$. pilosa em fase reprodutiva, ocorrentes em áreas ruderais, nos arredores da cidade de Botucatu, SP, foram coletados no período de março a setembro de 1993. Seis coletas foram realizadas com intervalo de cerca de 30 dias, sendo amostrados 10 indivíduos que apresentavam distribuição agrupada e 5 indivíduos isolados. Consideraramse plantas agrupadas aquelas em que os ramos de indivíduos vizinhos se tocavam, formando uma mancha com um número variável de plantas; plantas isoladas eram aquelas estabelecidas distantes de vizinhos conspecíficos no mínimo $15 \mathrm{~m}$.

Após a realização da coleta da parte aérea das plantas, as seguintes características foram avaliadas: altura; número de ramos secundários; número de capítulos imaturos, maduros sem predação e predados, distribuição de matéria seca no caule e nos capítulos em diferentes estágios de desenvolvimento. Os capítulos predados eram identificados visualmente pois apresentavam aquênios maduros retidos na infrutescência que, após a maturação não se abre como em capítulos maduros não predados.

Os dados referentes às características vegetativas e reprodutivas foram submetidos ao teste de correlação de Spearman (Sokal \& Rohlf, 1981), dentro de cada tipo de agrupamento. A 
comparação entre os dados obtidos para os dois grupos de plantas foi realizada pela prova não paramétrica de Mann-Whitney (Siegel, 1975).

\section{RESULTADOS E DISCUSSÃO}

Os dados de correlação entre as características vegetativas e reprodutivas para cada tipo de agrupamento encontram-se na Tabela 1 (plantas agrupadas) e na Tabela 2 (plantas isoladas). Observa-se, em ambas as Tabelas, que houve grande ocorrência de correlações positivas, destacando-se a correlação entre a altura da planta e a matéria seca do caule. Estes resultados indicam que a altura é um parâmetro adequado para avaliação do tamanho das plantas podendo ser utilizada em substituição a matéria seca que é geralmente o mais indicado para esta finalidade (Hutchings, 1997). A utilização da altura apresenta vantagens metodológicas pois é uma medida mais fácil de se obter, sendo ainda uma técnica não destrutiva a qual pode ser requerida em diferentes circunstâncias.

A distribuição de freqüência de classes de altura obtida para plantas agrupadas e isoladas encontram-se nas Figuras 1 e 2, respectivamente. No grupo de plantas agrupadas, os limites mínimo e máximo de altura foram 15 e $160 \mathrm{~cm}$, respectivamente; as maiores freqüências se localizaram entre as classes que abrangem valores de 15 a $77 \mathrm{~cm}$. Em relação às plantas isoladas, o limite mínimo foi de $19 \mathrm{~cm}$ e o máximo foi de 151 $\mathrm{cm}$. No intervalo de 19 a $63 \mathrm{~cm}$ se localizaram as classes de maior frequiência. A ocorrência de plantas de diferentes alturas, conforme demonstrada nas Figuras 1 e 2, resulta numa estrutura de tamanho assimétrica refletindo, possivelmente, as diferentes características e oportunidades de crescimento experimentadas pelos indivíduos (Hutchings, 1997). Em geral, as populações vegetais estão constituídas por pequeno número de plantas grandes e numerosas plantas menores (Weiner \& Solbrig, 1984) e este também é o caso da população estudada pois a maior frequiência ocorreu nas classes de menor tamanho.

O potencial reprodutivo das plantas também pode ser inferido, nas condições deste estudo, pelo valor de altura, haja vista a correlação positiva e significativa entre estes parâmetros (Tabelas 1 e 2). Plantas maiores produzem, portanto, maior número e biomassa de capítulos. Quanto à incidência de predação, verifica-se que, para ambas as situações, houve igualmente correlação positiva e significativa com a altura das plantas. Na Figura 3 pode-se observar esta tendência, embora deva ser ressaltada a ocorrência de nível similar de predação em plantas de diferentes alturas. A intensidade de predação parece não sofrer variação acentuada em plantas de até cerca de $1 \mathrm{~m}$ de altura; somente a partir deste tamanho, foram encontradas plantas com um número consideravelmente maior de capítulos predados. Estes resultados parecem indicar que plantas grandes tendem a sustentar maior número de capítulos atacados e a estes resultados aparentemente se aplicariam as explicações enunciadas pela Hipótese do Vigor (Price, 1991). Porém, a preferência por plantas mais vigorosas deve ser considerada com restrições no caso de $B$. pilosa, uma vez que plantas de até cerca de $1 \mathrm{~m}$ de altura também apresentaram capítulos predados, embora em menor quantidade.

Em relação ao grau de agrupamento, observou-se que plantas isoladas e agrupadas não diferiram significativamente em relação a grande maioria das características analisadas (Tabela 3). Desta forma, observa-se que o nível de agrupamento não interferiu na intensidade da predação, evidenciando que a distribuição agrupada não condiciona altos níveis de ataque aos capítulos. Assim, B. pilosa, nas condições analisadas, não confirma a Hipótese de Concentração de Recursos.

Os dados obtidos no presente trabalho permitem concluir que maiores níveis de predação tendem a ocorrer em plantas mais vigorosas, independente do grau de agrupamento dos indivíduos. 
TABELA 1. Análise de correlação entre características biométricas vegetativas e reprodutivas de plantas agrupadas de Bidens pilosa.

\begin{tabular}{|c|c|c|c|c|c|c|c|c|c|c|c|}
\hline Variáveis & 1 & 2 & 3 & 4 & 5 & 6 & 7 & 8 & 9 & 10 & 11 \\
\hline No. ramos secundários (1) & ", & $0,48 * *$ & $0,54 * *$ & 0,08 & $0,59 * *$ & $0,52 * *$ & $0,64 * *$ & $0,42 * *$ & $0,60 * *$ & 0,13 & $0,69 * *$ \\
\hline No. capítulos imaturos (2) & , & , & 0,09 & $-0,03$ & $0,27 *$ & 0,04 & 0,18 & $0,93 * *$ & 0,09 & 0,14 & $0,39 * *$ \\
\hline No. capítulos maduros (3) & ", & , &, & $0,34 * *$ & $0,97 * *$ & $0,71 * *$ & $0,78 * *$ & $-0,03$ & $0,75^{* *}$ & 0,16 & $0,69 * *$ \\
\hline No. capítulos predados (4) & , & , & , & , & $\mathbf{0 , 4 3 * *}$ & $0,31 *$ & 0,27 & $-0,09$ & 0,1 & $0,84 * *$ & 0,28 \\
\hline No. total capítulos (5) & , & , &, & , & , & $0,69 * *$ & $0,78 * *$ & 0,13 & $0,71 * *$ & $0,29 *$ & $0,74 * *$ \\
\hline Altura (6) & $"$ & , & , & , & , & , & $\mathbf{0 , 8 3 * *}$ & $-0,02$ & $0,75^{* *}$ & $0,30^{*}$ & $0,73 * *$ \\
\hline Matéria seca do caule (7) & " & , & , & , & , & , & 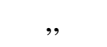 & 0,15 & $0,85^{* *}$ & 0,22 & $0,84 * *$ \\
\hline Matéria seca imatura (8) & ", & , & , & ", & " & " & " & " & $-0,02$ & 0,07 & $0,30 *$ \\
\hline Matéria seca capítulo maduro (9) & , &, & , & , & , &, & , &, & , & 0,16 & $0,90 * *$ \\
\hline Matéria seca capítulo predado (10) & " &, & , & , & , & , & , & , & , & " & $0,43 * *$ \\
\hline Matéria seca total capítulos (11) & , & , & , & , & , & , &, & , & , & , & , \\
\hline
\end{tabular}

TABELA 2. Análise de correlação entre características biométricas vegetativas e reprodutivas de plantas isoladas de Bidens pilosa.

\begin{tabular}{|c|c|c|c|c|c|c|c|c|c|c|c|}
\hline Variáveis & 1 & 2 & 3 & 4 & 5 & 6 & 7 & 8 & 9 & 10 & 11 \\
\hline No. ramos secundários (1) & .. & 0,16 & $0,69^{* *}$ & 0,12 & $0,55^{* *}$ & $0,48^{*}$ & $0,62 * *$ & $0,50 * *$ & $0,53 * *$ & 0,18 & $0,59 * *$ \\
\hline No. capítulos imaturos (2) & .. & .. & $\mathbf{0 , 1 7}$ & $-0,02$ & $0,68^{* *}$ & 0,22 & 0,25 & $0,43 *$ & 0,26 & 0,01 & 0,36 \\
\hline No. capítulos maduros (3) & .. & .. &.. & $0,49 * *$ & $0,81 * *$ & $0,74 * *$ & $0,70 * *$ & $0,55^{* *}$ & $0,92 * *$ & $0,55^{* *}$ & $0,82^{* *}$ \\
\hline No. capítulos predados (4) & .. & .. & .. & .. & $0,47^{*}$ & $0,44^{*}$ & 0,22 & 0,18 & $0,45^{*}$ & $0,95^{* *}$ & 0,32 \\
\hline No. total capítulos (5) & .. & .. & .. & .. & .. & $0,67 * *$ & $0,63 * *$ & $0,63 * *$ & $0,81 * *$ & $0,52 * *$ & $0,78 * *$ \\
\hline Altura (6) & .. & .. & .. & .. & .. & .. & $0,84 * *$ & $0,79 * *$ & $0,73 * *$ & $0,50 * *$ & $0,86^{* *}$ \\
\hline Matéria seca do caule (7) & .. & .. & .. & .. & .. & .. & .. & $\mathbf{0 , 8 3 * *}$ & $0,65^{* *}$ & 0,25 & $0,79^{* *}$ \\
\hline Matéria seca imatura (8) & .. & .. & .. & .. & .. & .. & .. & .. & $\mathbf{0 , 5 8 * *}$ & 0,24 & $0,85 * *$ \\
\hline Matéria seca capítulo maduro (9) & .. & .. & .. & .. & .. & .. & .. & .. & .. & $\mathbf{0 , 5 1 * *}$ & $0,90 * *$ \\
\hline Matéria seca capítulo predado (10) & .. & .. & .. & .. & .. & .. & .. & .. & .. & .. & $\mathbf{0 , 3 9}$ \\
\hline Matéria seca total capítulos (11) & .. & .. & .. & .. & .. & .. & .. & .. & .. & .. & .. \\
\hline
\end{tabular}




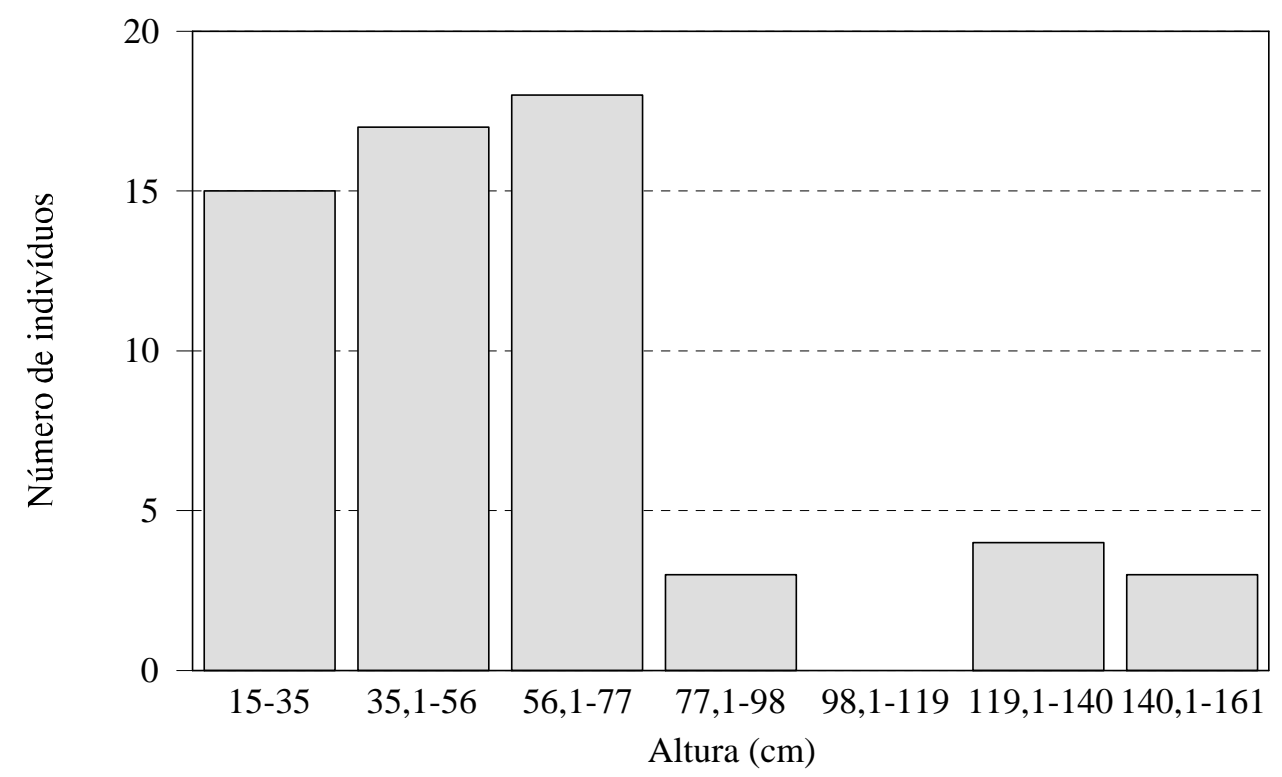

FIGURA 1. Distribuição da frequiência de classes de altura em plantas agrupadas de Bidens pilosa.

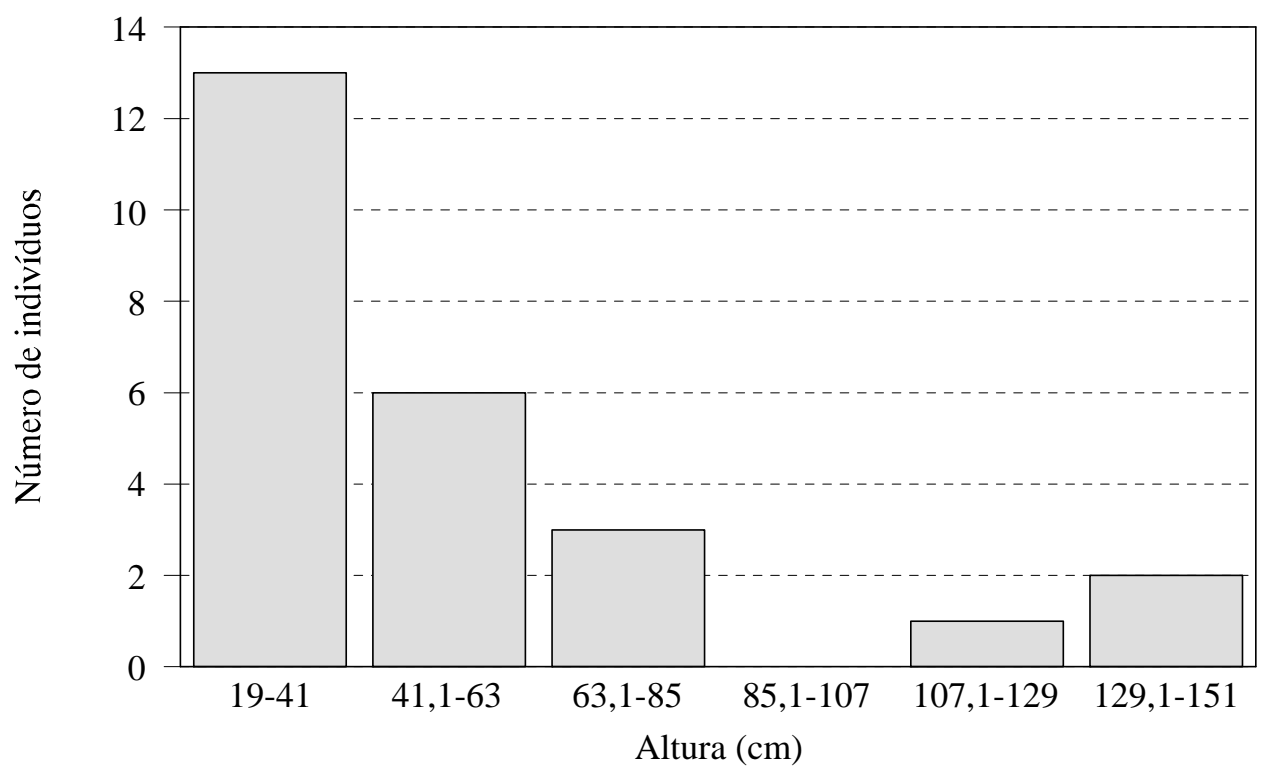

FIGURA 2. Distribuição da frequiência de classes de altura em plantas isoladas de Bidens pilosa. 


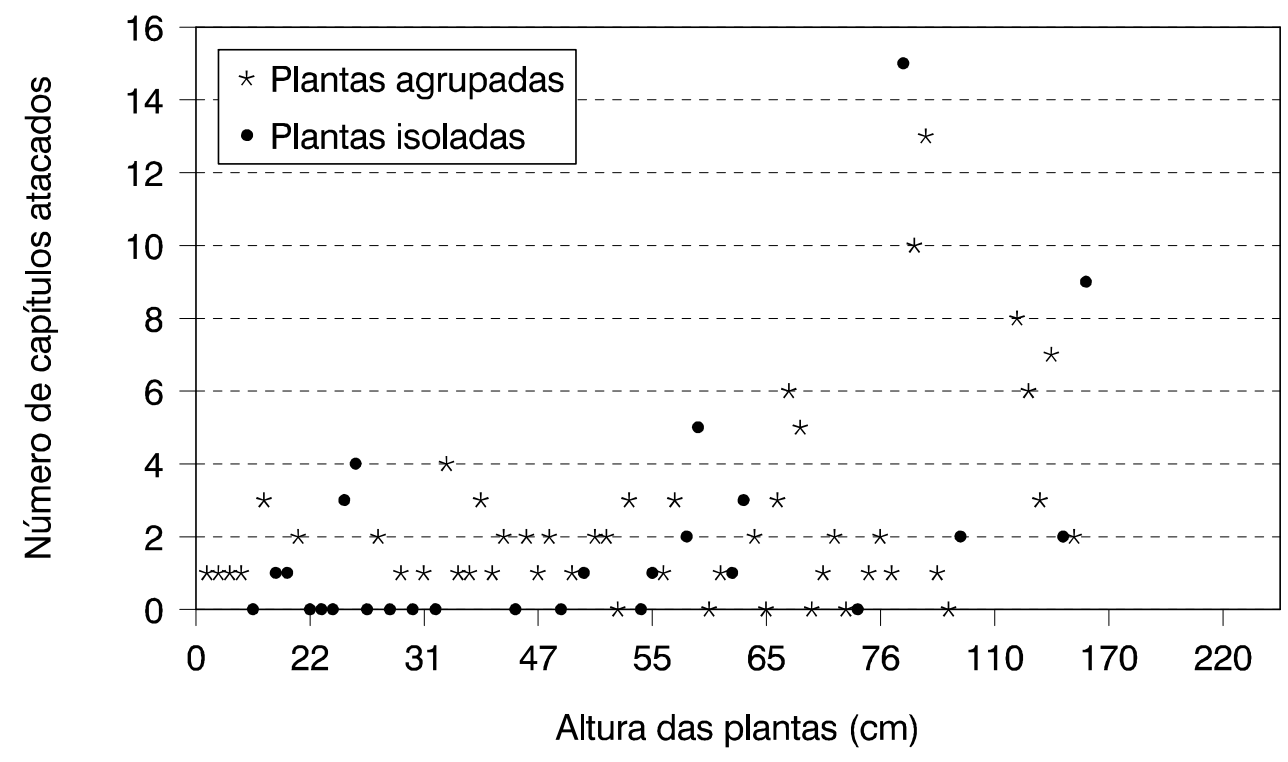

FIGURA 3. Relação entre o número de capítulos predados e altura $(\mathrm{cm})$ de Bidens pilosa.

TABELA 3. Valores das medianas e dos postos médios das estruturas vegetativas e reprodutivas de Bidens pilosa: plantas agrupadas $(\mathrm{G})$; plantas isoladas (I); número (No.); matéria seca (MS).

\begin{tabular}{lcccc}
\hline Variáveis & $\mathrm{G}$ & $\mathrm{I}$ & Estatística $\mathrm{z}$ & Nível significância \\
\hline No. ramos secundários & $3(41,18) \mathrm{a}$ & $4(47,38) \mathrm{a}$ & 1.07 & $\mathrm{p}>0,10$ \\
No. capítulos imaturos & $2(39,27) \mathrm{a}$ & $4(51,96) \mathrm{b}$ & 2.19 & $\mathrm{p}<0,05$ \\
No.capítulos maduros & $9,5(45,66) \mathrm{a}$ & $7(36,62) \mathrm{a}$ & 1.54 & $\mathrm{p}>0,10$ \\
No.capítulos maduros & $1(45,37) \mathrm{a}$ & $1(37,30) \mathrm{a}$ & 1.42 & $\mathrm{p}>0,10$ \\
No. total capítulos & $14,5(43,38) \mathrm{a}$ & $12(41,84) \mathrm{a}$ & 0.28 & $\mathrm{p}>0,10$ \\
Altura & $53,5(46,15 \mathrm{a}$ & $35(35,50) \mathrm{a}$ & 1.89 & $0,05<\mathrm{p}<0,10$ \\
MS caule & $1,1(44,54) \mathrm{a}$ & $0,8(39,32) \mathrm{a}$ & 0.89 & $\mathrm{p}>0,10$ \\
MS capítulos imaturos & $0,04(39,62) \mathrm{a}$ & $0,1(51,12) \mathrm{b}$ & 1.97 & $\mathrm{p}<0,05$ \\
MS capítulos maduros & $0.07(45,60) \mathrm{a}$ & $0,04(36,76) \mathrm{a}$ & 1.5 & $\mathrm{p}>0,10$ \\
MS capítulos predados & $0,04(45,98) \mathrm{a}$ & $0,04(35,84) \mathrm{a}$ & 1.76 & $0,05<\mathrm{p}<0,10$ \\
MS total capítulos & $0,1(45,27) \mathrm{a}$ & $0,1(37,56) \mathrm{a}$ & 1.31 & $\mathrm{p}>0,10$ \\
\hline
\end{tabular}

Quanto à intensidade de predação observou-se a ocorrência de pequena proporção de capítulos predados por planta (Tabela 3), evidenciando que no caso em estudo, a predação de sementes não se constitui num fator de regulação da densidade populacional.

O potencial de utilização dos dípteros predadores das sementes de B. pilosa em programas de controle biológico apresenta, desta forma, restrições pois seria muito pouco eficiente e, por se tratar de um sistema planta/herbívoro específico, apresenta ação duvidosa em programas de manejo integrado, conforme salientado por Pitelli (1982). 


\section{LITERATURA CITADA}

ALTIERI, M. A. A ecologia dos herbívoros tropicais em agroecossistemas de policultivo. In: SIMPÓSIO INTERNACIONAL SOBRE ECOLOGIA EVOLUTIVA DE HERBÍVOROS TROPICAIS, 1988, Campinas. Anais...Campinas: Universidade Estadual de Campinas, 1988. p.69-70.

BEGON, M., HARPER, J.L., TOWNSEND, C.R. Ecology: individuals, populations and communities. 3ed. Oxford: Blackwell Science, 1996. p.174.

CRAWLEY, M. J. Plant-herbivore dynamics. In: Crawley, M. J (ed.). Plant ecology, 2. ed., Oxford, Blackwell Science, 1997. p.401474.

HARPER, J.L. Population biology of plants. Oxford: London Academic Press, 1977. 812p.

HUTCHINGS, The structure of plant population. In: CRAWLEY, M.J. (ed.). Plant ecology. 2.ed., Oxford, Blackwell Science, 1997. p. 325-358

LEITÃO FILHO, H. F., ARANHA, C., BACCHI, $O$. Plantas invasoras de culturas no estado de São Paulo. São Paulo: HUCITEC/AGIPLAN, 1975. v.2, p. 292597.

LEWINSOHN, T. M. Insects in flower heads of Asteraceae in southeast Brazil: a case study on tropical species richness. In: PRICE, P.W. et al. (eds): Plant aninal interactions: evolutionary ecology in tropical and temperate regions. New York: John Wiley \& Sons, 1991. p.525-559.
LORENZI, H. Plantas daninhas do Brasil. Nova Odessa, Editora Plantarum, 1982. 425p.

NEDER, L.T.C. Avaliação dos tipos morfológicos de aquênios e da fauna presente em capítulos de $B$. pilosa (Asteraceae). Botucatu: UNESP, 1983. 28p. Monografia (Bacharelado em Ciências Biológicas) - Instituto de Biociênicas, Universidade Estadual Paulista, 1983.

NEEDHAN, J. G. An insect community lives in flower heads. Nat. Geogr., v.90, p.340$356,1946$.

PAGLIARINI, A. C. Levantamento florístico de comunidades de plantas daninhas ocorrentes no município de Botucatu-SP. Botucatu: UNESP, 1991. 46p. Monografia (Bacharelado em Ciências Biológicas) Instituto de Biociênicas, Universidade Estadual Paulista, 1991.

PITELLI, R. A. Manejo integrado de plantas daninhas. In: MARCONDES, D. A. S. (Ed.). Controle integrado de plantas daninhas. São Paulo: CREAA, 1982. p. 27-41.

PRICE, P. W. The plant vigor hypothesis and herbivore attack. Oikos, v.62, p.244-251, 1991.

SIEGEL, S. Estatística não paramétrica. São Paulo: McGraw-Hill, 1975. 350p.

SOKAL, R. R.; ROHLF, F. J. Biometry; the principal and practice of statistics in biological research. 2.ed. New York: W. H. Freeman, 1981. 859p.

STEYSKAL, G. C. The dipterous fauna of the heads of B. pilosa. Flo. Ent., v.55, n.2, p.87-88, 1972. 
Yanagizawa et al.

WEINER, J.; SOLBRIG, T. O. The meaning and measurement of size hierarchies in plant populations. Oecologia, v.61, p.334-336, 1984.
WHITE, T.C.R. An index to measure weather induced stress of trees associated with outibreak of psyllids in Australia. Ecology, v.50, n.5, p.905-909, 1969. 